\title{
Prospective comparison of three predictive rules for assessing severity of community-acquired pneumonia in Hong Kong
}

\section{Shin Yan Man, Nelson Lee, Margaret Ip, Gregory E Antonio, Shirley SL Chau, Paulina Mak, Colin A Graham, Mingdong Zhang, Grace Lui, Paul K S Chan, Anil T Ahuja, David S Hui, Joseph J Y Sung, Timothy H Rainer}

Thorax 2007;62:348-353. doi: 10.1136/thx.2006.069740

\begin{abstract}
See end of article for authors' affiliations

Correspondence to: Professor T H Rainer, Accident and Emergency Medicine Academic Unit Chinese University of Hong Kong, Rooms 107/113 1 st Floor, Trauma and Emergency Centre, Prince of Wales Hospital, Shatin, NT, Hong Kong; thrainer@cuhk. edu.hk
\end{abstract}

Received 5 August 2006 Accepted 30 October 2006 Published Online First 22 November 2006
Background: Community-acquired pneumonia (CAP) is a leading infectious cause of death throughout the world, including Hong Kong.

Aim: To compare the ability of three validated prediction rules for CAP to predict mortality in Hong Kong: the 20 variable Pneumonia Severity Index (PSI), the 6-point CURB65 scale adopted by the British Thoracic Society and the simpler CRB65.

Methods: A prospective observational study of 1016 consecutive inpatients with CAP (583 men, mean (SD) age 72 (17) years) was performed in a university hospital in the New Territories of Hong Kong in 2004. The patients were classified into three risk groups (low, intermediate and high) according to each rule. The ability of the three rules to predict 30 day mortality was compared.

Results: The overall mortality and intensive care unit (ICU) admission rates were $8.6 \%$ and $4.0 \%$, respectively. PSI, CURB65 and CRB65 performed similarly, and the areas under the receiver operating characteristic (ROC) curve were $0.736(95 \% \mathrm{Cl} 0.687$ to 0.736$), 0.733$ (95\% $\mathrm{Cl} 0.679$ to 0.787 ) and 0.694 (95\% Cl 0.634 to 0.753$)$, respectively. All three rules had high negative predictive values but relatively low positive predictive values at all cut-off points. Larger proportions of patients were identified as low risk by PSI (47.2\%) and CURB65 (43.3\%) than by CRB65 (12.6\%).

Conclusion: All three predictive rules have a similar performance in predicting the severity of CAP, but CURB65 is more suitable than the other two for use in the emergency department because of its simplicity of application and ability to identify low-risk patients.
C mmunity-acquired pneumonia (CAP) is a leading infectious cause of death throughout the world ${ }^{1-3}$ includ-

ing Hong Kong, ${ }^{4}$ and a major drain on healthcare resources. ${ }^{5}$ The estimated annual costs for treating pneumonia in the USA and UK are US $\$ 8.4$ billion $^{6}$ and $£ 441$ million, ${ }^{7}$ respectively, with most of these costs being used for inpatient care. In Hong Kong the problem may be even worse since the outbreak of severe acute respiratory syndrome (SARS), ${ }^{8}$ with some emergency physicians adopting a conservative approach to the management of pneumonia by indiscriminately admitting nearly all patients with CAP into hospital.

Several international organisations have developed guidelines $^{7-11}$ or scoring systems ${ }^{12-14}$ in an attempt to stratify CAP according to risk severity; the aim is to enhance the appropriateness of admission and to lower unnecessary admission rates. The Pneumonia Severity Index (PSI) ${ }^{12}$ is a widely used predictive method in North America. It is a two-step scoring system using 20 variables and was developed for identifying low-risk patients or potential candidates for outpatient care. CURB ${ }^{13}{ }^{14}$ is a modified version of the British Thoracic Society (BTS) assessment tool which relies on four parameters for scoring-namely, mental confusion, blood urea level, respiratory rate and diastolic blood pressure. In $2003 \mathrm{Lim}_{\text {et }} \mathrm{al}^{14}$ added age $\geqslant 65$ years as a fifth prognostic variable to the CURB scoring system and turned it into a 6-point scoring scale (0-5) known as CURB65 which was adopted by the BTS as the new severity assessment strategy for CAP in 2004. ${ }^{15}$ A simpler model, the CRB65, without the requirement for laboratory investigations (urea), could be a useful tool outside hospital as it is based only on clinical parameters for scoring (confusion, respiratory rate, blood pressure, and age) and has comparable results to CURB65. ${ }^{14}$
Some centres have validated these guidelines in their own context, ${ }^{16-18}$ but others have reported that as many as $27 \%$ of patients with PSI class I-III (low risk) require admission to the ICU $^{19}$ and $40 \%$ of patients in low-risk groups were admitted to hospital. ${ }^{20}$ These rules have never been tested in Hong Kong, despite the high incidence of CAP and despite recent major outbreaks of atypical pneumonia such as SARS.

The aim of this study was to compare the effectiveness of these three validated prediction rules (PSI, CURB65 and CRB65) to predict mortality and to evaluate their potential application as a guide for admission or discharge from the emergency department. We investigated whether the null hypothesis that there is no difference between the risk stratification groups of the three rules for predicting 30 day mortality on admission can be rejected.

\section{METHODS}

\section{Study design and patients}

Data were collected prospectively from consecutive patients admitted to hospital through the emergency department with a provisional diagnosis of CAP between 1 January 2004 and 31 December 2004. Ethical approval was obtained from the Institutional Review Boards of the Hospital Authority of Hong Kong and of the Prince of Wales Hospital to conduct a prospective study in patients with CAP.

Abbreviations: CAP, community-acquired pneumonia; ICU, intensive care unit; LOS, length of stay; PSI, Pneumonia Severity Index; ROC curve, receiver operating characteristic curve 


\section{Setting}

The study was conducted in the emergency department of the Prince of Wales Hospital, the main teaching hospital of the Faculty of Medicine of the Chinese University of Hong Kong. The hospital has over 1200 beds, a workforce of 3400 healthcare staff and serves a population of 1.5 million people in the New Territories of Hong Kong. The emergency department sees 160000 new patients per annum and admits $24 \%$ to hospital. Approximately 50 patients are admitted to internal medicine wards each day, of which $20 \%$ are admitted with suspected infective causes.

\section{Definitions and CAP scoring systems}

CAP was defined in our study as an acute infection of the pulmonary parenchyma that was associated with symptoms of acute infection, accompanied by the presence of an acute infiltrate on a chest radiograph in a patient not hospitalised for more than 14 days before onset of symptoms. ${ }^{10}$ All patients were assessed by a specialist emergency physician before admission and by a specialist in respiratory medicine or infectious diseases before discharge. The final diagnosis was made by a respiratory physician and was based on the clinical, radiological and laboratory results. Patients with CAP were stratified into low, intermediate and high-risk groups according to the PSI, CURB65 and CRB65 scoring systems. ${ }^{12}{ }^{14}$ The rules were then compared for their ability to predict 30 day all-cause mortality.

\section{Inclusion and exclusion criteria}

All patients aged more than 17 years, admitted to hospital and who fulfilled the definition of CAP were included in the study. Exclusion criteria were patients with severe immunosuppression, defined as HIV infection, neutropenia $<1 \times 10^{9} / 1$, on longterm immunosuppressants or steroids, or solid organ transplant recipients. Patients with a final diagnosis of pulmonary tuberculosis were excluded because of the different disease progress, management and prognosis. The period for this study did not include the SARS outbreak of 2003, and there were no isolated cases in Hong Kong during 2004. We also excluded patients who had been in hospital within the previous 14 days and those with a diagnosis other than CAP after admission.

\section{Radiographic evaluation}

All patients had a frontal chest radiograph in the emergency department. Images were assessed using a picture archiving and communication system (PACS), viewer workstation with a $2048 \times 2048$ pixel monitor (Magicview Version VA22E; Siemens, Munich, Germany). The images were reviewed by senior radiologists working in pairs and interpretation was by consensus. Radiologists reviewed the $x$ rays of all patients and were blind to the clinical information except that they were aware that this was a study aiming to include patients with CAP.

\section{Data collection}

All data were recorded according to a standard questionnaire by a trained research nurse. Data collected at admission to the emergency department included age, gender, admission from home or a nursing home, coexisting illness, symptoms and clinical parameters: blood pressure, pulse rate, respiratory rate, percutaneous oxygen saturation, tympanic temperature and mental confusion (defined in our study as Glasgow Coma Scale $\leqslant 14$ ). Additional data collected for all patients either in the emergency department or immediately after admission included laboratory results (complete blood count, arterial blood gas, glucose, electrolytes and urea), radiographic findings reported by the radiologists and outcome variables (the requirement for ICU admission or mechanical ventilation, length of stay (LOS) in hospital and all-cause 30 day mortality).

\section{Outcome measures}

The primary outcome measure was 30 day all-cause mortality. Secondary outcome measures included ICU admission rate, LOS in hospital and ICU-free days (defined in our study as days alive between day 1 and day 30 which were spent outside the ICU).

\section{Statistical analysis}

All data were analysed using SPSS Version 13.0 for Windows and Statview for Windows (SAS Institute Version 5.0). Descriptive statistics of demographic and clinical variables included frequencies, percentage, means and standard deviation (SD). One-way ANOVA and Kruskal-Wallis tests were used for comparisons of normal and skewed continuous variables of more than two groups, respectively. Sensitivity, specificity, positive predictive value, negative predictive value and the areas beneath the receiver operating characteristic (ROC) curves for predicting 30 day mortality in each predictive rule were compared. ROC curves were generated first for the population as a whole and then with nursing home residents excluded. For all analyses a two tailed $\mathrm{p}$ value of $<0.05$ was considered statistically significant.

Table 1 Baseline characteristics and outcome measures of patients with pneumonia $(\mathrm{N}=1016)$

\begin{tabular}{|c|c|}
\hline Baseline characteristics & Mean (SD), range or $n(\%)$ \\
\hline Age & 72 (7.2), 17-103 \\
\hline Male, n (\%) & $583(57.4 \%)$ \\
\hline Nursing home, $n(\%)$ & $247(24.3 \%)$ \\
\hline \multicolumn{2}{|l|}{ Coexisting illness } \\
\hline Congestive heart failure, $\mathrm{n}(\%)$ & $124(12.2 \%)$ \\
\hline COPD, n (\%) & $167(16.4 \%)$ \\
\hline Bronchiectasis, n (\%) & $22(2.2 \%)$ \\
\hline Asthma, $\mathrm{n}(\%)$ & $50(4.9 \%)$ \\
\hline Pneumoconiosis, n (\%) & $2(0.2 \%)$ \\
\hline Old pulmonary tuberculosis, n (\%) & $85(8.4 \%)$ \\
\hline Cerebrovascular disease, $\mathrm{n}(\%)$ & $176(17.3 \%)$ \\
\hline Neoplastic disease, n (\%) & $1(0.1 \%)$ \\
\hline Renal disease, $\mathrm{n}(\%)$ & $84(8.3 \%)$ \\
\hline Liver disease, $\mathrm{n}(\%)$ & $13(1.3 \%)$ \\
\hline \multicolumn{2}{|l|}{ Clinical parameters } \\
\hline Pulse rate & 101 (19.9), 43-161 \\
\hline Systolic blood pressure & $142(28.2), 65-247$ \\
\hline Diastolic blood pressure & $72(15.5), 40-140$ \\
\hline Temperature & $38(1.1), 34-42$ \\
\hline Respiratory rate & $25(5.8), 14-48$ \\
\hline Glasgow Coma Score & $15(0.8), 3-15$ \\
\hline $\mathrm{O}_{2}$ saturation $\left(\mathrm{SaO}_{2} \%\right)$ & $95(4.4), 70-100$ \\
\hline \multicolumn{2}{|l|}{ Laboratory results } \\
\hline Sodium (mmol/l) & $136.0(6.1), 111-264$ \\
\hline Urea (mmol/l) & $7.9(5.9), 1.1-53$ \\
\hline Glucose (mmol/l) & $7.3(2.4), 0.3-24.6$ \\
\hline Arterial $\mathrm{pH}$ & $7.4(0.06), 6.6-7.6$ \\
\hline Haematocrit & $0.35(0.06), 0.14-0.71$ \\
\hline \multicolumn{2}{|l|}{ Radiographic findings } \\
\hline Bilateral lung involvement, n (\%) & $280(27.6 \%)$ \\
\hline$>2$ zones involvementt, $\mathrm{n}(\%)$ & $276(27.2 \%)$ \\
\hline Pleural effusion, $\mathrm{n}(\%)$ & $99(9.7 \%)$ \\
\hline \multicolumn{2}{|l|}{ Outcome measures } \\
\hline 30 day mortality, n (\%) & $87(8.6 \%)$ \\
\hline ICU admission, $\mathrm{n}(\%)$ & $41(4.0 \%)$ \\
\hline Mechanical ventilation, $\mathrm{n}(\%)$ & $25(2.5 \%)$ \\
\hline Hospital LOS (days) & $9.3(8.5)$ \\
\hline
\end{tabular}

COPD, chronic obstructive pulmonary disease; ICU, intensive care unit; LOS length of stay.

*All data are means (SD) unless stated otherwise.

TThe lungs were divided artificially into six zones in the radiograph: right and left upper, middle and lower zones. 
Table 2 Distribution of patients and 30 day mortality in each risk class of predictive rules*

\begin{tabular}{lll}
\hline Risk groups & $\begin{array}{l}\text { Number of patients } \\
(\mathbf{N}=1016)\end{array}$ & $\begin{array}{l}30 \text { day mortality } \\
(\mathbf{N}=87)\end{array}$ \\
\hline PSI class & & $0(0)$ \\
I & $0(0)$ & $2(0.8)$ \\
II & $242(23.8)$ & $12(5)$ \\
III & $238(23.4)$ & $33(9.3)$ \\
IV & $355(34.9)$ & $40(22.1)$ \\
V & $181(17.8)$ & $<0.001$ \\
p valuet & & $1(0.9)$ \\
CURB65 & $107(10.5)$ & $12(3.6)$ \\
0 & $333(32.8)$ & $23(7.3)$ \\
1 & $315(31)$ & $31(16.4)$ \\
2 & $189(18.6)$ & $17(26.6)$ \\
3 & $64(6.3)$ & $3(37.5)$ \\
4 & $8(0.8)$ & $<0.001$ \\
5 & & $3(2.3)$ \\
P value† & & $25(5.1)$ \\
CRB65 & $128(12.6)$ & $33(11.2)$ \\
0 & $489(48.1)$ & $22(23.2)$ \\
1 & $294(28.9)$ & $4(40)$ \\
2 & $95(9.4)$ & $<0.001$ \\
3 & $10(1)$ & \\
4 & & \\
p value† & & \\
\hline
\end{tabular}

*All data are numbers (\%) unless stated otherwise.

tp value $\left(\chi^{2}\right.$ test for 30 day mortality).

\section{RESULTS}

Of 1648 consecutive patients admitted with a provisional diagnosis of CAP, 1016 (62\%) were included in the study; 632 patients (38\%) were excluded either because they had exclusion criteria or a non-CAP diagnosis. The baseline characteristics of the 1016 patients included in the study are shown in table 1: $583(57.4 \%)$ were male and the mean (SD) age was 72 (17) years, $789(77.7 \%)$ were aged $>65$ years and $247(24.3 \%)$ were nursing home residents. All patients were treated with empirical antibiotics according to the standard hospital guideline of the Hospital Authority of Hong Kong. ${ }^{21}$

\section{Outcome}

Eighty-seven patients (8.6\%) died within 30 days of admission, $41(4 \%)$ needed ICU care and $25(2.5 \%)$ needed mechanical ventilation. The mean (SD) LOS in hospital was 9.3 (8.5) days.

\section{Comparisons of mortality and ICU admission rate}

Table 2 shows the patient distribution and 30 day mortality in each risk score of the predictive rules. The different PSI risk classes and CURB65/CRB65 scores were categorised into low, intermediate and high-risk groups (table 3 ) according to their original study methodology. ${ }^{13}$ All three predictive rules showed the same trend of increasing mortality with worsening risk groups $(p<0.001)$. The mortality rate of low-risk groups was $2.9 \%$ in PSI, $3.0 \%$ in CURB65 and $2.3 \%$ in CRB65. PSI and CURB65 classified a significantly larger proportion of patients (47.2\% and $43.3 \%$, respectively) as low risk than CRB65 $(12.6 \%)$

ICU admission rates also increased with the risk levels of each rule, but were only statistically significant in CURB65 and CRB65 ( $p=0.02$ and $p=0.01$, respectively). The ICU admission rate of low-risk groups was $2.7 \%$ in PSI, $2.3 \%$ in CURB65 and $3.9 \%$ in CRB65. The mortality rate of high-risk patients was $22.1 \%$ in PSI, $19.5 \%$ in CURB65 and $24.8 \%$ in CRB65. The ICU admission rate was $9.5 \%$ in the high-risk group of CRB65, which was higher than PSI and CURB65 (6.6\% and 6.5\%, respectively). The calculated sensitivity and specificity for the high-risk group of each rule in identifying ICU admission were $29.3 \%$ and $82.7 \%$ for the PSI, $41.5 \%$ and $75.0 \%$ for CURB 65 and $24.4 \%$ and $90.3 \%$ for CRB65. A separate analysis using a modified American Thoracic Society (ATS) rule ${ }^{22}$ was performed in this cohort. Four hundred and thirty-six patients $(42.9 \%)$ were classified as having severe CAP and $8.5 \%$ of them needed intensive care. The sensitivity and specificity for identifying patients needing intensive care were $90.2 \%$ and $59.1 \%$, respectively.

\section{Comparisons of LOS in hospital and ICU-free days}

The in-hospital LOS increased with increasing risk group, while the number of ICU-free days decreased with increasing severity (table 3). The differences in LOS and ICU-free days in different risk groups were all statistically significant.

\section{Comparison of predictive accuracy}

Table 4 shows the sensitivity, specificity, positive and negative predictive values for 30 day mortality at different cut-off points for each scoring system. PSI had higher sensitivities and lower specificities than CURB65/CRB65 at all different risk levels. The sensitivities of low risk cut-off points were $83.9 \%$ in PSI, $85.1 \%$ in CURB65 and $96.6 \%$ in CRB65. All three prediction rules had

Table 3 Comparison of different outcome measures of predictive rules*

\begin{tabular}{|c|c|c|c|c|c|}
\hline Risk groups & $\begin{array}{l}\text { No of patients } \\
(\mathrm{N}=1016)\end{array}$ & $\begin{array}{l}30 \text { day mortality } \\
(\mathrm{N}=87)\end{array}$ & $\begin{array}{l}\text { ICU admission } \\
(\mathrm{N}=41)\end{array}$ & LOS in hospital & ICU-free days \\
\hline \multicolumn{6}{|l|}{ PSI class } \\
\hline Low (II-III) & $480(47.2)$ & $14(2.9)$ & $13(2.7)$ & $6(5.0-9.8)$ & $29.6(3.0)$ \\
\hline Intermediate (IV) & $355(34.9)$ & $33(9.3)$ & $16(4.5)$ & $7(5.0-10.0)$ & $28.7(7.9)$ \\
\hline High (V) & $181(17.8)$ & $40(22.1)$ & $12(6.6)$ & $8(5.5-12.0)$ & $25.0(9.9)$ \\
\hline$p$ Valuet & & $<0.001$ & 0.063 & $<0.001$ & $<0.001$ \\
\hline \multicolumn{6}{|l|}{ CURB65 } \\
\hline Low (score 0-1) & $440(43.3)$ & $13(3.0)$ & $10(2.3)$ & $6(5.0-9.0)$ & $29.7(4.5)$ \\
\hline Intermediate (score 2) & $315(31.0)$ & $23(7.3)$ & $14(4.4)$ & $7(5.0-10.0)$ & $28.6(5.8)$ \\
\hline High (score 3-5) & $261(25.7)$ & $51(19.5)$ & $17(6.5)$ & $8(5.0-12.5)$ & $26.2(9.9)$ \\
\hline$p$ Valuet & & $<0.001$ & 0.020 & $<0.001$ & $<0.001$ \\
\hline \multicolumn{6}{|l|}{ CRB65 } \\
\hline Low (score 0 ) & $128(12.6)$ & $3(2.3)$ & $5(3.9)$ & $6(4.3-9.8)$ & $28.8(1.6)$ \\
\hline Intermediate (score 1-2) & $783(77.0)$ & $58(7.4)$ & $26(3.3)$ & $7(5.0-10.0)$ & $28.8(6.3)$ \\
\hline High (score 3-4) & 105 (10.3) & $26(24.8)$ & $10(9.5)$ & $8(5.0-13.0)$ & $24.5(11.3)$ \\
\hline p Valuet & & $<0.001$ & 0.010 & 0.007 & $<0.001$ \\
\hline
\end{tabular}

*All data are numbers (\%) unless stated otherwise.

tp Value (Kruskal-Wallis test for LOS in hospital, ANOVA test for ICU-free days, $\chi^{2}$ test for 30 day mortality and ICU admission). \pm Median $\left(25-75^{\text {th }}\right.$ interquartile range).

§Mean (SD). 
Table 4 Sensitivity, specificity, positive and negative predictive values of 30 day mortality of the different predictive rules*

\begin{tabular}{|c|c|c|c|c|}
\hline Cut-off points & Sensitivity & Specificity & Positive predictive value & Negative predictive value \\
\hline \multicolumn{5}{|l|}{ PSI risk class } \\
\hline$\geqslant 1$ & NA & NA & NA & NA \\
\hline$\geqslant \|$ & 100 & 0.0 & 8.6 & NA \\
\hline$\geqslant I I I$ & 97.7 & 25.8 & 11.0 & 99.2 \\
\hline$\geqslant \mathrm{IV} \dagger$ & 83.9 & 50.2 & 13.6 & 97.1 \\
\hline V & 46.0 & 84.8 & 22.1 & 94.4 \\
\hline \multicolumn{5}{|l|}{ CURB65 score } \\
\hline$\geqslant 0$ & 100 & 0.0 & 8.6 & NA \\
\hline$\geqslant 1$ & 98.9 & 11.4 & 9.5 & 99.1 \\
\hline$\geqslant 2 \dagger$ & 85.1 & 46.0 & 12.8 & 97.0 \\
\hline$\geqslant 3$ & 58.6 & 77.4 & 19.5 & 95.2 \\
\hline$\geqslant 4$ & 23.0 & 94.4 & 27.8 & 92.9 \\
\hline 5 & 3.4 & 99.5 & 37.5 & 91.7 \\
\hline \multicolumn{5}{|l|}{ CRB65 scores } \\
\hline$\geqslant 0$ & 100 & 0.0 & 8.6 & NA \\
\hline$\geqslant 1 \dagger$ & 96.6 & 13.5 & 9.5 & 97.7 \\
\hline$\geqslant 2$ & 67.8 & 63.4 & 14.8 & 95.5 \\
\hline$\geqslant 3$ & 29.9 & 91.5 & 24.8 & 93.3 \\
\hline 4 & 4.6 & 99.4 & 40.0 & 91.7 \\
\hline
\end{tabular}

${ }^{*}$ All numbers are percentages

tCut-off points accepted as threshold to define low-risk group according to original study design. ${ }^{11} 13$

high negative predictive values but low positive predictive values at all cut-off points.

The ROC curves for 30 day mortality for each prediction scoring method are shown in fig 1 . There was no significant difference in the area under the ROC curves for each of the three scoring methods. When nursing home residents were excluded, the area under the ROC curves, which are not shown in fig 1 , for the remaining patients were 0.728 (95\% CI 0.662 to 0.793 ), 0.713 (95\% CI 0.639 to 0.788 ) and 0.654 (95\% CI 0.572 to 0.736 ) for PSI, CURB65 and CRB65, respectively.

\section{DISCUSSION}

Prediction rules may be useful adjuncts for clinical decision making. This study shows no clinical difference in performance between PSI, CURB65 and CRB65 for a large Hong Kong population.

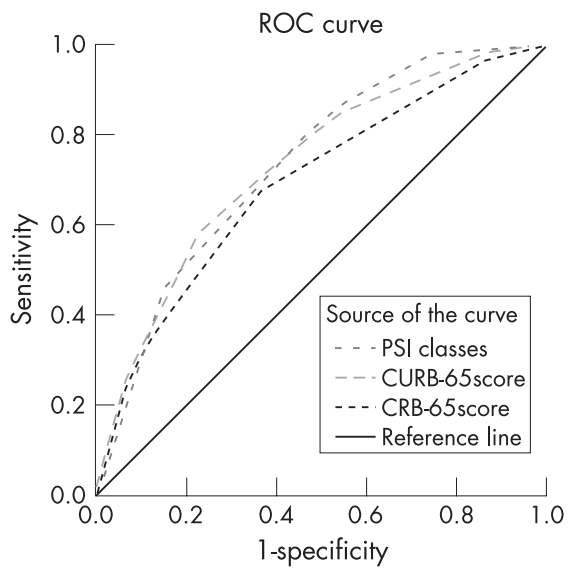

Area under the curve

\begin{tabular}{|c|c|c|c|}
\hline $\begin{array}{c}\text { Test result } \\
\text { variable(s) }\end{array}$ & Area & $95 \%$ confidence interval & p-value \\
\hline PSI classes & 0.736 & $0.687-0.786$ & $<0.001$ \\
\hline CURB-65score & 0.733 & $0.689-0.787$ & $<0.001$ \\
\hline CRB-65score & 0.694 & $0.634-0.753$ & $<0.001$ \\
\hline
\end{tabular}

Figure 1 Receiver operating characteristic (ROC) curves of different predictive rules in 30 day mortality.
More than three-quarters of the patients in our study were over 65 years old, reflecting the fact that most patients with CAP requiring hospital management are elderly. The management of this group is often challenging: they are more likely to have atypical clinical presentations, a different distribution of aetiological agents, coexisting illness, poor social support and a higher mortality rate. ${ }^{23}$ Emergency physicians in Hong Kong tend to adopt a conservative approach, admitting patients from this group into hospital without regard for their disease severity, especially in the absence of a good clinical guideline. This leads to many potentially unnecessary hospital admissions which not only strains hospital resources but also exposes some patients to increased risks of nosocomial infection.

The mortality rate in our study is comparable to those reported worldwide. ${ }^{71}$ A certain proportion of admitted patients could be considered for outpatient care, and a good predictive rule is needed to help frontline emergency department staff. Similar to the findings in other studies, all three prediction rules had high negative predictive values but low positive predictive values for 30 day mortality at all cut-off points and are therefore more useful in ruling out serious illness. ${ }^{13} 1416$

The rules may be used to identify low-risk patients and guide emergency department discharge policies. The difference in mortality between low-risk groups is small, but more than $40 \%$ of the patients with CAP were identified as low risk by PSI and CURB65, meaning that at least $40 \%$ of the admitted patients could potentially have been considered for outpatient management using PSI and CURB65. However, only $12.6 \%$ of patients were identified as low risk by CRB65. PSI and CURB65 are therefore more useful than CRB65 for identifying potential patients with CAP for potential outpatient management. CURB65 is more practical than PSI if simplicity is taken into consideration. For identifying high-risk patients, all three rules have a low positive predictive value which makes them less useful in guiding decision making for inpatient management.

None of the three clinical decision rules appeared to be useful for identifying patients requiring ICU care because of their low sensitivities. When the most recent parameters for the ATS rule were applied to our study population, the sensitivity was higher (90\% vs $78 \%$ ) but specificity lower $(59.1 \%$ vs $94 \%$ ) than the original study. ${ }^{22}$ This suggests that the ATS rule, when applied in our setting, may be more useful as a "rule out" than a "rule in" tool. No prospective studies have clearly related disease 
severity with ICU admission rates. Generally, patients of higher risk classes have higher rates of ICU admission. However, unlike 30 day mortality, the association between them is not well defined. In fact, the ICU admission rate in our study was low compared with previous work ${ }^{17}$ (4\% vs $16.7 \%$ ) despite similar mortality rates in both studies. It is clear that the criteria for ICU admission vary from country to country and from hospital to hospital, and disease severity is not the only factor to consider. Other factors such as disease prognosis, pre-morbid status, age of patient, and the availability of ICU resources are all routinely considered by intensive care physicians before admitting a patient to the ICU.

Our study comprised mainly elderly patients who may have more coexisting illness, poorer quality of life and poorer prognoses than younger patients; these patients may have a lower chance of receiving intensive care than the general population. Indeed, the 30 day mortality rate in our study was higher than the ICU admission rate ( $8.6 \%$ vs $4 \%)$, meaning that at least some patients with severe disease died without prior ICU care. Prediction rules are therefore not useful in predicting ICU admission, especially among elderly patients, although they give an indication of disease severity.

The relationship between ICU-free days and disease severity may be better than the relationship between ICU days or LOS in hospital and disease severity as patients with more severe illness may die earlier and have a short LOS in hospital. The inhospital LOS and ICU-free days depend on the time needed to reach clinical stability, which is closely related to the severity of the illness. ${ }^{24}$ High-risk patients need more time to reach clinical stability, and hence have longer stays in hospital and fewer ICU-free days.

Although the characteristics of nursing home residents with pneumonia may be different from patients with $\mathrm{CAP}^{25}$ in practice emergency physicians are unlikely to differentiate between these two groups. Assessment of prognosis will depend upon physiological factors rather than the differentiation of nursing home versus non-nursing home residents. Although nosocomial pneumonia and CAP may have different bacteriological aetiologies, when our database was analysed with and without their inclusion, there was no significant difference in predictive ability or risk stratification. Therefore, for the purpose of this study, we included these residents in our analysis.

The strength of this study lies in its prospective design, large sample size, the completeness of data collection and a tertiary teaching hospital setting with a wide catchment area. All radiographs were reported by senior radiologists who were blinded to the disease conditions of the patients, giving rise to less potential bias in radiological interpretation. A further strength is that current clinical policy in our institution is to obtain arterial blood gas measurements from all patients admitted with CAP irrespective of their oxygen saturation level.

One limitation of our study is the sample selection-namely, all enrolled patients were inpatients admitted through the emergency department with a diagnosis of CAP. These patients were, on average, more severely ill and more advanced in age. PSI, CURB65, CRB65 and most other predictive rules were not originally designed for use in elderly patients, and this may account for the overall lower accuracy of the three predictive rules in our study compared with other validation studies. ${ }^{16} 18$ The "confusion" variable used in our study was not the same as in the definition of CURB65. The definition of confusion for CURB65 was an Abbreviated Mental Test Score of $\leqslant 8$ or new disorientation to person, place and time. ${ }^{13}$ However, for our study, we defined confusion as a Glasgow Coma Scale score of $\leqslant 14$; many different dialects are used in Hong Kong, especially among the elderly which makes it impossible to use the Abbreviated Mental Test Score to screen our participants.
All predictive rules serve only as a guide to clinical management, and severity of illness is not the only factor which should be considered when deciding on whether or not to admit a patient. Social and home circumstances must be considered fully in reaching a clinical management decision, especially with elderly patients. Physicians should always exercise clinical judgment and common sense in making these sometimes difficult decisions.

In conclusion, no significant differences were found between PSI, CURB65 and CRB65 in predicting 30 day mortality. CURB65 may be more useful than PSI and CRB65 in a busy emergency department because of its simplicity in application and its ability to identify a reasonable proportion of low-risk patients for potential outpatient care.

\section{Authors' affiliations}

Shin Yan Man, Paulina Mak, Colin A Graham, Timothy H Rainer, Accident and Emergency Medicine Academic Unit, Chinese University of Hong Kong, Prince of Wales Hospital, Shatin, NT, Hong Kong

Nelson Lee, Grace Lui, David S Hui, Joseph J Y Sung, Department of Medicine and Therapeutics, Chinese University of Hong Kong, Prince of Wales Hospital, Shatin, NT, Hong Kong

Margaret lp, Shirley S L Chau, Paul K S Chan, Department of

Microbiology, Chinese University of Hong Kong, Prince of Wales Hospital, Shatin, NT, Hong Kong

Gregory E Antonio, Anil T Ahuja, Department of Diagnostic Radiology and Organ Imaging, Chinese University of Hong Kong, Prince of Wales Hospital, Shatin, NT, Hong Kong

Mingdong Zhang, Center for Emerging Infectious Diseases, Faculty of Medicine, Chinese University of Hong Kong, Prince of Wales Hospital, Shatin, NT, Hong Kong

Funding and support: The authors received no outside funding or support. Competing interests: None declared.

\section{REFERENCES}

1 Woodhead M. Community-acquired pneumonia: severity of illness evaluation Infect Dis Clin North Am 2004; 18:791-807.

2 World Health Organization. WHO Statistical Information System (WHOSIS). WHO Mortality Database 2005.

3 US Department of Health and Human Services. Centers for Disease Control and Prevention. National Center for Health Statistics. Chartbook on trends in the health of Americans. DHHS publication No. 2005-1232. 2005.

4 Department of Health, Government of the Hong Kong Special Administrative Region. Annual Report 2003/2004. A8: Number of deaths by ten leading causes of death by sex by age group 2003.

5 Colice GL, Morley MA, Asche C, et al. Treatment costs of community-acquired pneumonia in an employed population. Chest 2004;125:2140-5.

6 Niederman MS, McCombs JS, Unger AN, et al. The cost of treating communityacquired pneumonia. Clin Ther 1998;20:820-37.

7 British Thoracic Society Standards of Care Committee. BTS guidelines for the management of community acquired pneumonia in adults. Thorax 2001;56(Suppl 4):IV1-64

8 Rainer TH, Cameron PA, Smit D, et al. Evaluation of WHO criteria for identifying patients with severe acute respiratory syndrome out of hospital: prospective observational study. BMJ 2003;326:1354-8.

9 Mandell LA, Marrie TJ, Grossman RF, et al. Canadian guidelines for the initial management of community-acquired pneumonia: an evidence-based update by the Canadian Infectious Diseases Society and the Canadian Thoracic Society. The Canadian Community-Acquired Pneumonia Working Group. Clin Infect Dis 2000;31:383-421

10 Bartlett JG, Dowell SF, Mandell LA, et al. Practice guidelines for the management of community-acquired pneumonia in adults. Infectious Diseases Society of America. Clin Infect Dis 2000;31:347-82.

11 Niederman MS, Mandell LA, Anzueto A, et al. American Thoracic Society guidelines for the management of adults with community-acquired pneumonia. Diagnosis, assessment of severity, antimicrobial therapy, and prevention. Am J Respir Crit Care Med 2001;163:1730-54.

12 Fine MJ, Auble TE, Yealy DM, et al. A prediction rule to identify low-risk patients with community-acquired pneumonia. N Engl J Med 1997;336:243-50.

13 Neill AM, Martin IR, Weir R, et al. Community acquired pneumonia: aetiology and usefulness of severity criteria on admission. Thorax 1996;51:1010-6.

14 Lim WS, van der Eerden MM, Laing R, et al. Defining community acquired pneumonia severity on presentation to hospital: an international derivation and validation study. Thorax 2003;58:377-82.

15 Macfarlane JT, Boldy D. 2004 update of BTS pneumonia guidelines: what's new? Thorax 2004;59:364-6. 
16 Aujesky D, Auble TE, Yealy DM, et al. Prospective comparison of three validated prediction rules for prognosis in community-acquired pneumonia. Am J Med 2005; 1 18:384-92.

17 Ewig S, de Roux A, Bauer T, et al. Validation of predictive rules and indices of severity for community acquired pneumonia. Thorax 2004;59:421-7.

18 Capelastegui A, Espana PP, Quintana JM, et al. Validation of a predictive rule for the management of community-acquired pneumonia. Eur Respir $J$ 2006;27:151-7.

19 Angus DC, Marrie TJ, Obrosky DS, et al. Severe community-acquired pneumonia: use of intensive care services and evaluation of American and British Thoracic Society diagnostic criteria. Am J Respir Crit Care Med 2002; 166:717-23.

20 Roson B, Carratala J, Dorca J, et al. Etiology, reasons for hospitalization, risk classes, and outcomes of community-acquired pneumonia in patients hospitalized on the basis of conventional admission criteria. Clin Infect Dis 2001;33:158-65.

21 Anon. Reducing bacterial resistance with IMPACT - Interhospital Multidisciplinary Programme on Antimicrobial Chemotherapy, 2nd ed.Version 2.2, 2003.

22 Ewig S, Ruiz M, Mensa J, et al. Severe community-acquired pneumonia. Assessment of severity criteria. Am J Respir Crit Care Med 1998;158:1 102-8. 23 Janssens JP, Krause KH. Pneumonia in the very old. Lancet Infect Dis 2004:4: 112-24.

24 Halm EA, Fine MJ, Marrie TJ, et al. Time to clinical stability in patients hospitalized with community-acquired pneumonia: implications for practice guidelines. JAMA 1998;279:1452-7.

25 Lim WS, Macfarlane JT. A prospective comparison of nursing home acquired pneumonia with community acquired pneumonia. Eur Respir J 2001;18:362-8. 